eCommons@AKU

January 1993

\title{
Molecular characterization of neurotransmitter transporters
}

Saad Shafqat

Aga Khan University, saad.shafqat@aku.edu

M Velaz- Faircloth

A Guadaño- -Ferraz

R T Fremeau

Follow this and additional works at: http://ecommons.aku.edu/pakistan_fhs_mc_med_neurol

\section{Recommended Citation}

Shafqat, S., Faircloth, M., -Ferraz, A., Fremeau, R. (1993). Molecular characterization of neurotransmitter transporters. Molecular Endocrinology, 7(12), 1517-1529.

Available at: http://ecommons.aku.edu/pakistan_fhs_mc_med_neurol/114 


\title{
Molecular Characterization of Neurotransmitter Transporters
}

\author{
Saad Shafqat, Maria Velaz-Faircloth, Ana Guadaño-Ferraz, and \\ Robert T. Fremeau, Jr. \\ Departments of Pharmacology and Neurobiology \\ Duke University Medical Center \\ Durham, North Carolina 27710
}

\section{INTRODUCTION}

Rapid chemical signaling between neurons and target cells is dependent upon the precise control of the concentration and duration of neurotransmitters in synaptic spaces. After transmitter release from activated nerve terminals, the principal mechanism involved in the rapid clearance from the synapse of the biogenic amine and amino acid neurotransmitters is active transport of the transmitter back into presynaptic nerve terminals or glial surrounding cells by one of a large number of specific, pharmacologically distinguishable membrane transport proteins (1). High affinity, $\mathrm{Na}^{+}$-dependent uptake activities, analogous to the noradrenergic carrier first described at peripheral synapses by Axelrod and colleagues (2), have been identified in mammalian central nervous system (CNS) nerve terminals for the biogenic amine neurotransmitters including norepinephrine (NE), dopamine (DA), and serotonin (5HT), for the excitatory amino acid neurotransmitters L-glutamate and L-aspartate, and for the inhibitory amino acid neurotransmitters $\gamma$-amino butyric acid (GABA) and glycine (3). The primary function of plasma membrane neurotransmitter transporters is to clear the synapse of the neurotransmitter between nerve impulses and to replenish neurotransmitter levels in presynaptic nerve terminals. In certain cases, $\mathrm{Na}^{+}$-dependent transport processes may also mediate the presynaptic accumulation of neurotransmitter precursors as well. For example, the rate-limiting step in the biosynthesis of acetylcholine appears to be $\mathrm{Na}^{+}-$dependent choline uptake into cholinergic nerve terminals by a hemicholiniumsensitive high affinity choline transporter (5).

Neurotransmitter transporters represent critical targets for therapeutic and pathological alterations of synaptic function. This is particularly apparent with the tricyclic antidepressants, amphetamines, and cocaine, agents that block biogenic amine transport and produce dramatic behavioral changes (6). Abnormalities of neurotransmitter transport can contribute to neuropathol-

0888-8809/93/1517-1529\$03.00/0

Molecular Endocrinology

Copyright 1993 by The Endocrine Society ogical processes. For example, blockade and/or reversal of the high affinity plasma membrane L-glutamate transporter during ischemia or anoxia elevates the extracellular concentration of L-glutamate to neurotoxic levels resulting in nerve cell damage (7). Conversely, specific GABA uptake inhibitors are being developed as potential anticonvulsant and antianxiety agents (8). The ability of synaptic transporters to accumulate certain neurotransmitter-like toxins, including $\mathrm{N}$-methyl-4phenylpyridine (MPP+), 6-hydroxydopamine, and 5,6dihydroxytryptamine, suggests a role for these active transport proteins in the selective vulnerability of neurons to exogenous agents (9). Therefore, a detailed understanding of the molecular structure, function, and regulation of neurotransmitter transporters will: 1) enhance our understanding of synaptic signaling, 2) elucidate the potential role of transporter defects in neurologic and psychiatric disorders; and 3) aid in the development of novel therapeutic agents. Finally, the discovery of novel synaptic transporters may contribute to the identification of new synaptic regulatory substances.

\section{TWO DISTINCT GENE FAMILIES ENCODE PLASMA MEMBRANE NEUROTRANSMITTER TRANSPORTERS}

Neurotransmitter transporters are representative members of a family of proteins that transduce free energy stored in an electrochemical gradient into work in the form of a concentration gradient. Active transport of neurotransmitters across the plasma membrane is driven by the transmembrane $\mathrm{Na}^{+}$gradient generated by the plasma membrane $\mathrm{Na}^{+} / \mathrm{K}^{+}$ATPase (reviewed in ref. 4). Additional ions are also required for transport of many neurotransmitters, including extracellular $\mathrm{Cl}^{-}$and intracellular $\mathrm{K}^{+}$. These ion sensitivities reflect cotransport of the ions with the neurotransmitter during each translocation cycle.

In recent years, molecular cloning studies have revealed that two distinct gene families code for plasma membrane neurotransmitter transporter proteins. 
These include: 1) the $\mathrm{Na}^{+}$- (and $\mathrm{Cl}^{-}$)-dependent family that includes transporters for GABA, NE, DA, 5HT, glycine, proline, taurine, betaine, and creatine; and 2) the $\mathrm{Na}^{+}$- (and $\mathrm{K}^{+}$)-dependent, but $\mathrm{Cl}^{-}$-independent, family of transporters that include transporters for the excitatory amino acids L-glutamate and L-aspartate (Fig. 1). These two families of plasma membrane transporters exhibit significant differences in their ionic requirements and proposed structures which are likely to reflect underlying differences in transport mechanisms. This review will discuss recent advances in our understanding of the structure, expression, and extent of the molecular diversity of these two neurotransmitter transporter gene families.

\section{$\mathrm{Na}^{+}-\left(\right.$and $\mathrm{Cl}^{-}$)-Dependent Neurotransmitter Transporters}

The molecular characterization of neurotransmitter transporters began with the pioneering work of Kanner

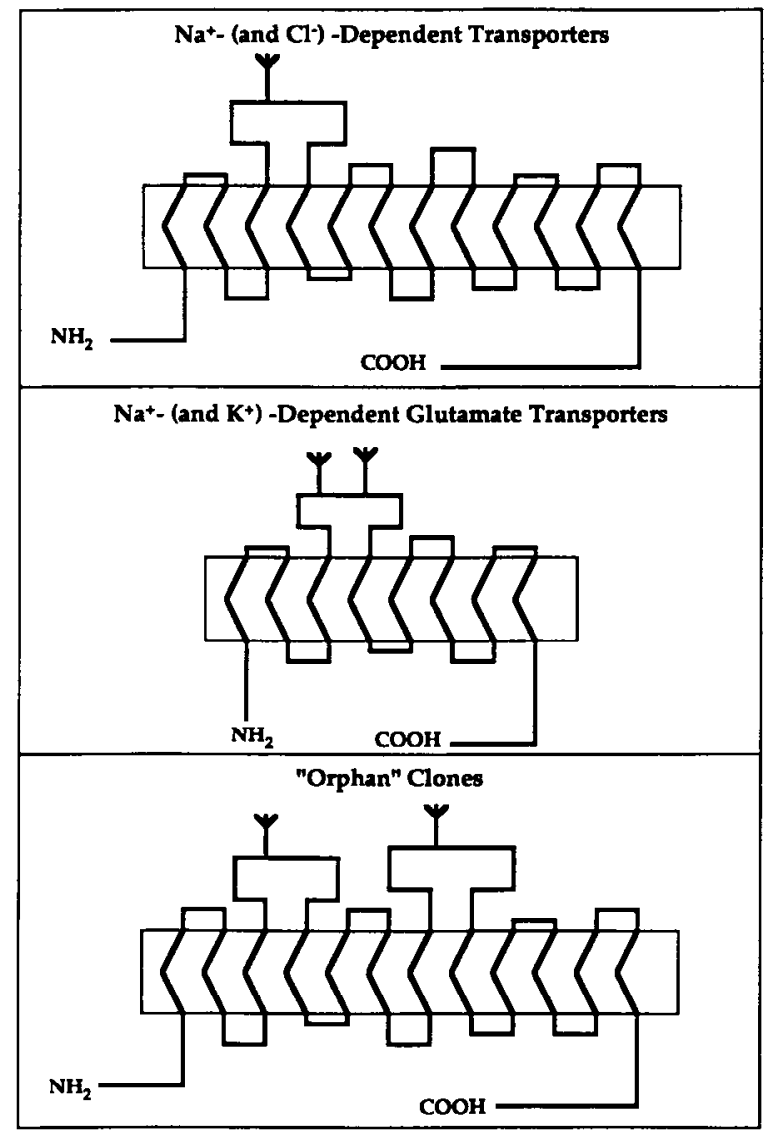

Fig. 1. Schematic models depicting the proposed transmembrane topology of the two major families of plasma membrane neurotransmitter transporters. Potential $\mathrm{N}$-linked glycosylation sites on the presumed extracellular domains are depicted. Due to the subjective nature of hydropathy analysis, the model proposed for the glutamate transporters is controversial (see text). Orphan clones refer to recently described putative transporters $(56,57)$ which bear sequence homology to the $\mathrm{Na}^{+}-$ (and $\mathrm{Cl}^{-}$--dependent transporters but exhibit a distinct inferred membrane topology. and colleagues $(10)$ on the purification and cloning of a high affinity $\mathrm{Na}^{+}$- (and $\mathrm{Cl}^{-}$)-dependent GABA transporter from rat brain (rGAT-1). Subsequently, Amara and colleagues (11) isolated a cDNA clone for a cocaineand antidepressant-sensitive human NE transporter (hNET) by expression cloning (11). Despite dramatic differences in pharmacological sensitivities to substrates and antagonists, rGAT-1 and hNET possessed significant $(46 \%)$ but dispersed amino acid sequence identities and exhibited similar inferred membrane topographies. No significant amino acid sequence identity was observed, however, between these two neurotransmitter transporters and other membrane transport proteins whose sequences had been determined, including the mammalian facilitated glucose transporters (12), the mammalian $\mathrm{Na}^{+} /$glucose cotransporter (13), the prokaryotic $\mathrm{Na}^{+}$-dependent cotransporters (14), or the ATP-binding cassette membrane transporters, including the multidrug resistance P-glycoproteins (15) and the cystic fibrosis transmembrane conductance regulator (16). These results established the presence of a distinct gene family of neurotransmitter transport proteins and paved the way for the rapid identification of additional members of this novel gene family.

Polymerase chain reaction (PCR) and low stringency screening techniques have been used to isolate additional members of the emerging family of $\mathrm{Na}^{+}$- (and $\mathrm{Cl}^{-}{ }^{-}$-dependent neurotransmitter transporters. Based upon amino acid sequence conservation (Fig. 2), these CDNAs can be divided into three subfamilies including: 1) the GABA, betaine, taurine, and creatine transporters; 2) the biogenic amine (NE, DA, and 5HT) transporters; and 3 ) the amino acid (L-proline and glycine) transporters. The observation that transporters for substrates with no known neurotransmitter role, such as betaine and creatine, belong to this family of transporters indicates that this family may subserve a greater diversity of physiological functions, and should more correctly be referred to as the $\mathrm{Na}^{+}$- (and $\mathrm{Cl}^{-}$)-dependent plasma membrane transporter family.

As depicted in Fig. 1, a tentative structural model for the $\mathrm{Na}^{+}$- (and $\mathrm{Cl}^{-}$)-dependent plasma membrane transporters predicts approximately 12 transmembrane $\alpha$ helical domains, cytoplasmic $\mathrm{NH}_{2}$ - and $\mathrm{COOH}$-termini, and a large, glycosylated, extracellular loop separating putative transmembrane domains 3 and 4 . This proposed topographical model remains to be experimentally verified. Although in each case a single cDNA is able to induce high affinity $\mathrm{Na}^{+}-$(and $\mathrm{Cl}^{-}$)-dependent transport in a nonneural cell line, the subunit stoichiometry of the native transporter complex has not been determined for any member of this family of membrane transport proteins.

\section{The Subfamily of GABA, Betaine, Taurine, and Creatine Transporters}

An exciting result to emerge from the molecular characterization of neurotransmitter transporters was the discovery that multiple transporter subtypes exist for 
certain neurotransmitters. Thus four distinct GABA transporter cDNAs have been described which exhibit $50-69 \%$ amino acid sequence identity but differ in their pharmacological properties and tissue expression patterns $(10,17-19,81)$. Traditionally, GABA transporters have been classified as "neuronal" or "glial" based upon their sensitivity to nipecotic acid (neuronal) or $\beta$-alanine (glial). In this respect, the first GABA transporter subtype cloned, rGAT-1 (10), exhibited a pharmacological profile consistent with that of a neuronal GABA transporter. Subsequent in situ hybridization and immunohistochemistry studies have confirmed that GAT- 1 is widely expressed in neurons throughout the nervous system. Interestingly, Clark and colleagues (17) have recently described a novel $\mathrm{Na}^{+}-$(and $\mathrm{Cl}^{-}$)-dependent GABA transporter, which they called GAT-B, that is potently inhibited by $\beta$-alanine. However, lesioning studies indicated that GAT-B is expressed by neurons within the mammalian brain (17). Thus the previously established pharmacological classification of GABA transporter subtypes is not supported by recent developments in the molecular characterization of GABA transporter subtypes.

At least two other GABA transporter subtypes have been identified, designated GAT-2 and GAT-3 by Liu and colleagues (19), that are expressed in both CNS and peripheral tissues including liver and kidney. One of these subtypes, GAT-3, is developmentally regulated in mouse brain (19). Its mRNA is abundant in neonatal but not adult brain. The physiological relevance of high affinity GABA transport in peripheral tissues such as liver and kidney remains to be determined.

The differential tissue distribution and distinct pharmacological properties of the four GABA transporter subtypes is consistent with the functional specialization of individual GABAergic synapses and/or cell types. Thus the potential exists for developing novel pharmacological agents which selectively modulate GABAergic neurotransmission at synapses that express a particular GABA transporter subtype. The preliminary pharmacological characterization of the four cloned GABA transporter subtypes represents an important first step in this direction (19).

Kanner and colleagues (20) have begun to identify specific amino acid residues which are important for the transport activity of rGAT-1. Because GABA is a zwitterionic molecule, and at least two $\mathrm{Na}^{+}$, and one $\mathrm{Cl}^{-}$, are cotransported with GABA during each translocation cycle, charged and polar amino acids located in the transmembrane-spanning domains are likely candidates to be important in substrate recognition and/or translocation (reviewed in Ref. 20). Indeed, Pantanowitz et al. (20) observed that mutation of an arginine residue in transmembrane domain 1 of rGAT-1, that is absolutely conserved throughout all the members of this family (see Fig. 3), abolished transport activity. The specificity of this effect was established by the observation that mutagenesis of four other highly conserved transmembrane "charged" amino acids resulted in mutant transporters with essentially "wild type" transport activity (20). Because the subfamily of biogenic amine transporters share this conserved arginine residue (see Fig. 3), but the cognate biogenic amine substrates lack the negatively charged carboxyl group present in $G A B A$, it is unlikely that this arginine residue plays a specific role in GABA transport, such as substrate recognition. More likely, it may participate in the binding of ions such as $\mathrm{Cl}^{-}$, or some other function shared by all the members of this family.

A canine betaine transporter (BGT) has been cloned from a Madin-Darby canine kidney (MDCK) cell cDNA library by expression cloning in Xenopus oocytes (21). BGT exhibits $88 \%$ amino acid sequence identity to the GABA transporter subtype designated GAT-2 (Fig. 2; $19,21)$. Betaine functions as a nonperturbing osmolyte in the renal medulla. Indeed, expression of BGT mRNA is induced by hypertonicity in cultured MDCK cells (21). Interestingly, BGT has a higher affinity for GABA (transport constant $=93 \mu \mathrm{M}$ ) than for betaine (transport constant $=398 \mu \mathrm{M}$ ), however, it is likely that only betaine is accumulated to significant levels in the renal medulla because plasma levels of betaine $(\sim 180 \mu \mathrm{M})$ far exceed those of GABA $(<1 \mu \mathrm{M})$ (reviewed in ref. 21). Northern blot analysis indicated that expression of BGT is localized to the kidney medulla; no expression was observed in kidney cortex, liver, ileal mucosa, epididymis, or brain (21). Thus although they exhibit striking amino acid sequence conservation, GAT-2 and BGT may not represent species homologs (mouse vs. dog) of the same transporter; in contrast to BGT, GAT-2 expression is readily detectable in mouse brain and liver, in addition to kidney (19).

Complementary DNAs encoding high affinity taurine transporters (TauT) have been recently isolated and functionally expressed from MDCK cells (22), rat brain (23), mouse brain (24), and human thyroid cells (25). Sequence similarities between the different clones indicate that they code for species homologs of the same taurine transporter. Like BGT, expression of TauT in MDCK cells is regulated by hypertonicity supporting the role of taurine as a nonperturbing osmolyte in the renal medulla (21). Unlike BGT, however, TauT is widely expressed in a number of tissues including brain, liver, heart, ileum, and epididymis, in addition to kidney (2225). Taurine (2-aminoethanesulfonic acid) is involved in a number of important physiological processes in mammals. These include: 1) bile acid conjugation in hepatocytes; 2) modulation of calcium flux and neural excitability; 3) detoxification; and 4) membrane stabilization (26). Taurine deficiency has been associated with retinal blindness, abnormalities in immune function, abnormalities in cardiac function, and reproductive abnormalities (26). The availability of TauT cDNA clones may help to clarify the physiological role of taurine in tissues such as retina and brain.

Recently, an $\mathrm{Na}^{+}$-dependent creatine transporter (CreaT) was cloned from rabbit brain and fast-twitch skeletal muscle (27). The pharmacological properties of CreaT are very similar to the endogenous creatine transport activity of COS-7 cells. Northern blot analysis 


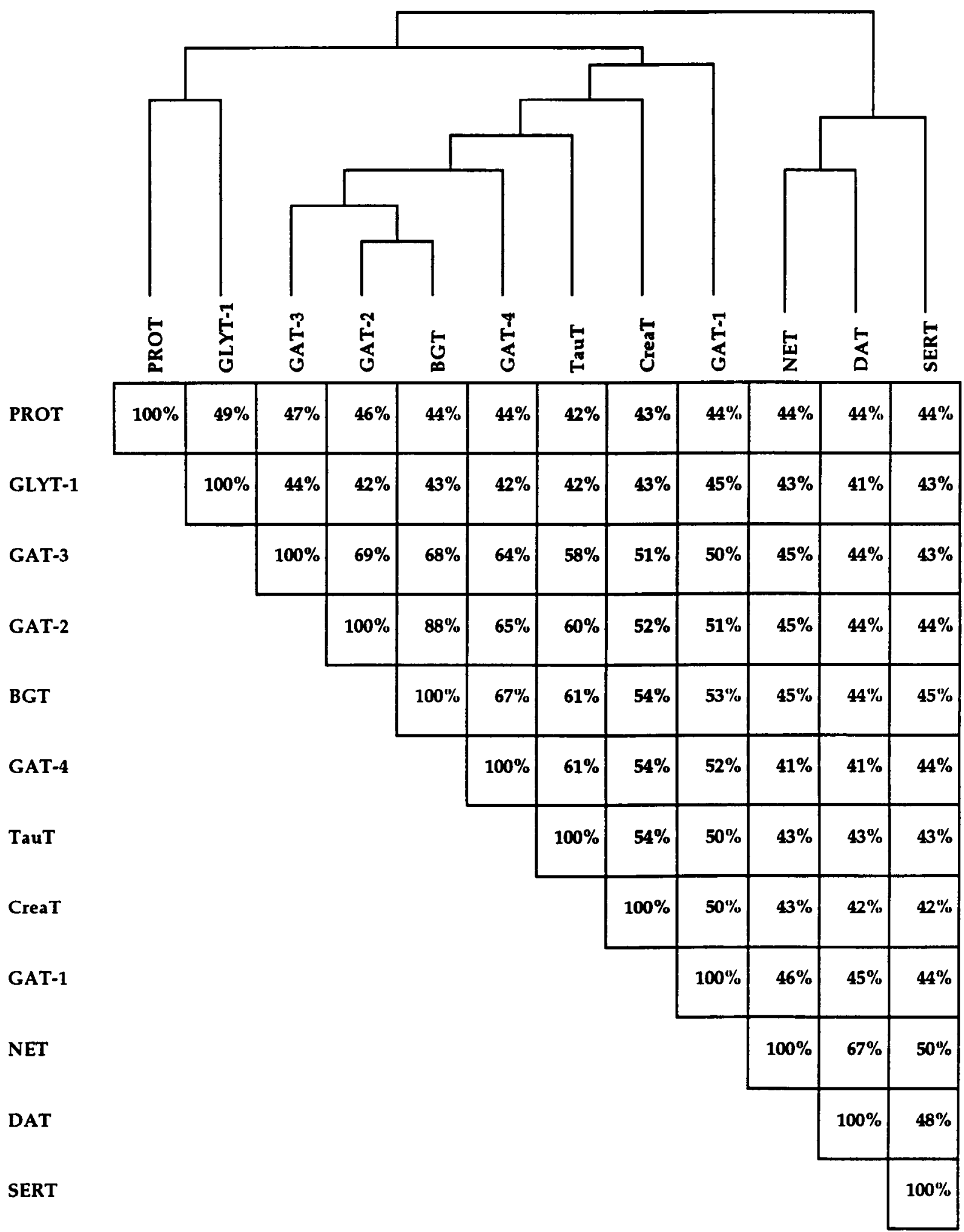

Fig. 2. Amino acid sequence relationships between various members of the $\mathrm{Na}^{+}$- (and $\mathrm{Cl}^{-}$)-dependent plasma membrane transporter gene family. The percent amino acid sequence identity between pairs of transporters is presented. As shown in the dendrogram, three distinct subfamilies can be resolved based upon amino acid sequence similarities: the subfamily of amino acid (L-proline and glycine) transporters; the subfamily of GABA, betaine, taurine, and creatine transporters; and the subfamily of biogenic amine transporters. For the various GABA transporter subtypes, to maintain consistency, we refer to the nomenclature of Liu et al. (19). PROT, High affinity rat brain L-proline transporter (51); GLYT-1, rat brain glycine transporter (47) [the sequence of GLYT-2, which is identical to GLYT-1 except for the first 15 amino acids (49) is not shown]; GAT-1 (10); GAT-2 (81), same as rat brain GAT-3 
revealed prominent expression of CreaT mRNA in tissues known to possess high creatine uptake capacity including kidney, heart, muscle, and brain (27). Active $\mathrm{Na}^{+}$-dependent uptake of creatine, and its interconversion with phosphocreatine, catalyzed by creatine kinase, is critical for ATP homeostasis. Preliminary in situ hybridization studies revealed a marked nonuniform distribution of CreaT mRNA in rat brain (28). High levels of labeling were observed over white matter fiber tracts including the corpus callosum and internal capsule, specific brain stem nuclei, and the granule cell layer of the cerebellum. Interestingly, Blakely and colleagues (28) have localized the human CreaT gene at, or near, a locus for an inherited juvenile onset CNS disease with demyelinating characteristics. The availability of the CreaT cDNA will now provide the opportunity to investigate the role of this transporter in individual tissues including the brain.

\section{The Subfamily of Biogenic Amine Transporters}

The cloning and functional expression of hNET from an SK-N-SH cell line (11) firmly established that a single polypeptide contained the binding sites for cocaine and antidepressants and induced high affinity NE transport in a nonneural cell (11). Sequence analysis of the hNET cDNA predicts a protein of 617 amino acids and a molecular size of approximately 69 kilodaltons. The hydropathy plot of hNET is superimposable with that of rGAT-1 indicating that these two proteins exhibit similar membrane topographies.

Northern blot analysis revealed that two distinct transcripts of 5.8 and 3.6 kilobases $(\mathrm{kb})$ hybridized to the hNET CDNA probe under high stringency conditions (11). The 5.6-kb message is abundantly expressed in SK-N-SH and PC-12 cells and in rat brain stem and adrenal gland. In contrast, the 3.6-kb RNA, which is likely to arise from the same gene, exhibits a more widespread and diffuse distribution (11). Thus the larger 5.6-kb message is likely to code for the NE transporter synthesized in noradrenergic neurons. The role of the 3.6-kb message remains to be determined.

The amino acid sequence homology between hNET and rGAT-1 provided the means for the isolation of rat $(29,30)$, bovine $(31)$, and human $(32,33)$ dopamine transporters (DATs) and rat $(34,35)$ and human (36) serotonin transporters (SERTs). The biogenic amine transporters exhibit 48-67\% amino acid sequence identity. This high degree of sequence conservation is not surprising based upon overlapping pharmacological sensitivities of these transporters to substrates and antagonists, including the psychostimulant cocaine. In situ hybridization studies have localized the expression of DAT and SERT to rat brain pathways specific to each of the cognate neurotransmitters. In particular, in situ hybridization studies using DAT CDNA revealed intense hybridization signals overlying the substantia nigra zona compacta, ventral tegmental area, periglomerular region of the olfactory bulb, and discrete regions of the hypothalamus, brain regions containing numerous dopaminergic cell bodies (39; Fremeau, Jr., R. T., M. G. Caron, and R. D. Blakely, unpublished observations). Similarly, SERT mRNA was observed overlying the serotonergic cells of the midbrain and brain stem Raphe nuclei (34). Interestingly, SERT mRNA was also detected in the rat adrenal gland (34). Because 5HT has been detected in mast cells lining rat adrenal arterioles (37), and in a population of adrenal medullary cells synthesizing epinephrine (38), these results support a physiological role for SERT in the adrenal gland.

The molecular cloning of hSERT from the human placental trophoblastic "JAR" cell line (36) has resolved previous discrepancies in the reported sequences of rat brain (34) and rat basophilic leukemia (RBL) cell line (35) SERTs. It is now clear that the rat brain and RBL SERT cDNAs are identical to each other and possess $92 \%$ amino acid identity with the human placental SERT. Thus it appears that peripheral and brain serotonin transporters are identical and arise from the same gene, providing further validation for studies of platelet serotonin transport activity in human neuropsychiatric disorders.

Previous studies suggest that second messengers may dynamically regulate the activity of the serotonin transporter. Myers and colleagues (40) observed that phorbol ester treatment of human pulmonary endothelial cells resulted in the rapid down-regulation of serotonin transport. This effect was antagonized by the protein kinase $\mathrm{C}$ inhibitor staurosporine. Interestingly, a comparison of the predicted amino acid sequences of the human and rat SERTs (36) revealed several conserved consensus sequences for protein kinasemediated protein phosphorylation. Thus future studies could examine the role that protein phosphorylation plays in the dynamic regulation of serotonin transport activity. Cool and colleagues (41) reported that treatment of placental JAR cells with cholera toxin resulted in a robust increase in serotonin transport activity. However, the relatively long time course of the effect compared to the rapid rise in CAMP levels, is more consistent with effects on gene expression or mRNA stability, rather than a direct effect on transport activity mediated by phosphorylation of the transporter protein. This is an important result because it provides a precedent for humoral modulation of the SERT gene. Such a mechanism could underlie the observed reductions in platelet and brain serotonin transporter sites and/or uptake in major depression, obsessive-cumpulsive dis-

described in ref. 18; GAT-3, originally called GAT-2 (18); GAT-4, originally designated GAT-B (17) (GAT-B was the second GABA transporter subtype identified, but for consistency of nomenclature we refer to it as GAT-4); BGT, canine betaine transporter (21); TauT, canine taurine transporter (22); CreaT, rabbit brain and skeletal muscle creatine transporter (27); NET, human norepinephrine transporter (11); DAT, rat brain dopamine transporter (29, 30); SERT, human serotonin transporter (36). 


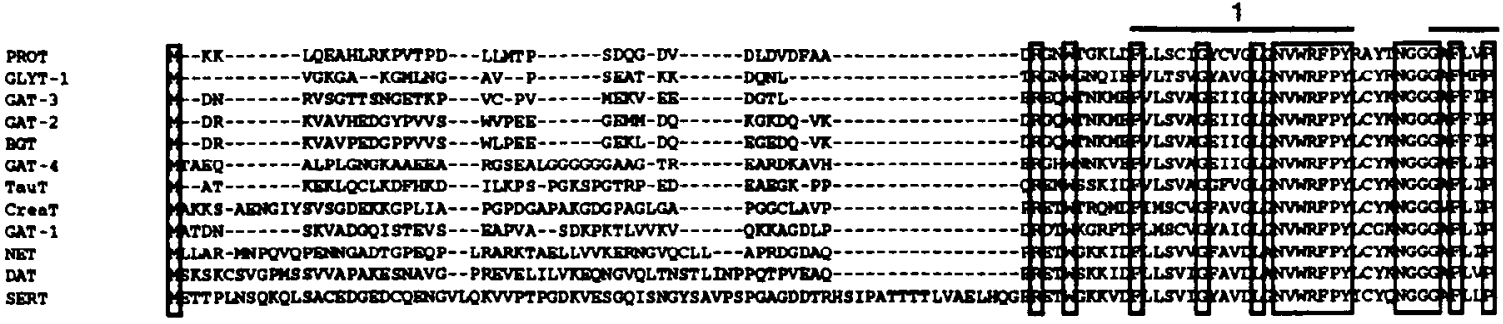

3

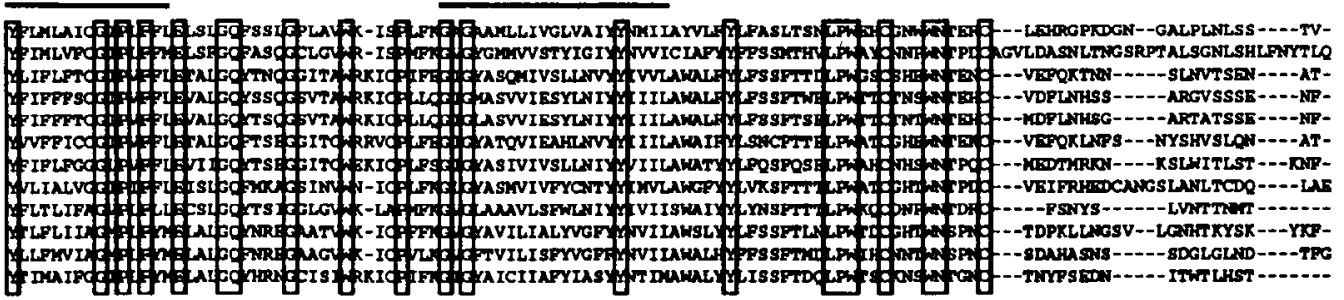

4

5
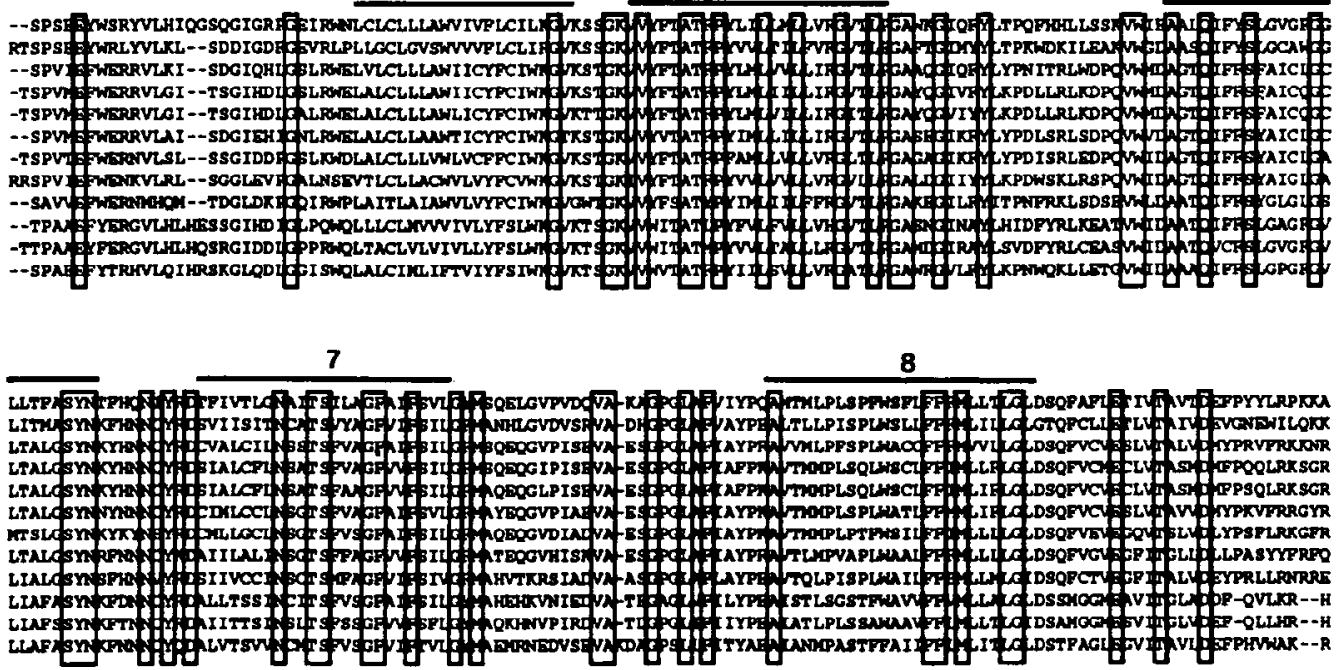

Fig. 3. Alignment of predicted amino acid sequences encoding different members of the $\mathrm{Na}^{+}$- (and $\mathrm{Cl}^{-}$)-dependent plasma membrane transporter gene family. Boxed residues represent those amino acids absolutely conserved across all the members of this family of transporters. Solid lines above PROT reflect the presumed location of the 12 transmembrane domains. For abbreviations see Fig. 2. 
order, or after suicide (reviewed in ref. 36). In this respect, it will be of considerable interest to examine the effects of circulating steroid hormones on SERT gene expression.

Two interesting features emerged from the pharmacological characterization of the cloned DATs. First, hDAT exhibited approximately 6-fold higher potency for cocaine compared to rDAT [inhibition constant $(\mathrm{Ki})=$ $58 \mathrm{~nm}$ for hDAT compared to $\mathrm{Kj}=320 \mathrm{nM}$ for rDAT] (32). In contrast, the potency of cocaine at hNET is Ki $=320 \mathrm{~nm}$ (11) and at hSERT it is $\mathrm{Ki}=610 \mathrm{~nm}$ (39). Thus, of the cloned human biogenic amine transporters, hDAT exhibits the highest affinity for cocaine supporting the "dopamine hypothesis" of cocaine reinforcement in humans. Second, there is an apparent discrepancy between the potency of the Parkinsonism-inducing neurotoxin, MPP ${ }^{+}$, to block DA uptake at hDAT or rDAT, and its potency to block the endogenous DA transport activity of rat striatal synaptosomes (32). Previously, Javitch and colleagues (42) observed that MPP+ inhibited DA transport in rat striatal synaptosomes with an $\mathrm{IC}_{50}$ of $170 \mathrm{nM}$. In contrast, Giros and colleagues (32) observed an $\mathrm{IC}_{50}$ value of approximately $10 \mu \mathrm{M}$ for $\mathrm{MPP}^{+}$in Ltk cells stably transfected with hDAT or rDAT. These results raise the possibility that cell lines transfected with DAT are missing a key component required for the recognition and/or transport of $\mathrm{MPP}^{+}$or, less likely, that a dopamine transporter subtype with a high affinity for $\mathrm{MPP}^{+}$may exist. Interestingly, Giros and colleagues (32) also observed an approximately 10-fold reduction in affinity of hDAT for DA itself, compared to rat striatal synaptosomes.

The human genes for the biogenic amine transporters have been mapped; however, no apparent linkage to any established disease gene loci have been observed. Human DAT has been mapped to the distal end of chromosome 5 (5p15.3) $(32,33)$; hSERT has been mapped to chromosome 17q11.1-17q12 (39); and hNET has been mapped to chromosome 16 (43).

The molecular cloning of the biogenic amine transporters provides important new tools for studying the biological basis of neuropsychiatric disorders. It should now be possible to examine whether defects in biogenic amine transporter genes are involved in specific brain disorders. In this respect, it is significant that Wright and colleagues (44) discovered that a point mutation in the $\mathrm{Na}^{+} /$glucose cotransporter gene abolishes its transport activity resulting in a potentially fatal human disease, glucose/galactose malabsorption. The availability of biogenic amine transporter cDNAs also provides the opportunity to use mutagenesis techniques to examine the structural basis of the differential drug sensitivities of these important synaptic regulatory proteins. For example, site-directed mutagenesis studies of rDAT are beginning to reveal amino acid residues important in cocaine binding and DA transport. These results suggest that like the catecholamine receptors, polar and charged residues within the putative transmembrane domains of DAT may comprise the substrate recognition site. Specifically, mutation of an aspartate residue in transmembrane domain 1 , which is unique to the biogenic amine transporters $\left(D_{79}\right.$ of DAT in Fig. 3 ), or two serine residues in transmembrane 7, disrupted DAT transport but differentially altered the binding of a cocaine analog (45). Thus it may be possible to dissociate amino acid residues required for substrate recognition vs. drug binding sites.

An intriguing study has recently been published demonstrating a dramatic decline in hDAT mRNA compared to tyrosine hydroxylase mRNA in the substantia nigra of aging humans (46). Since tyrosine hydroxylase is the rate-limiting enzyme in the biosynthesis of DA in dopaminergic neurons, this result implies that there is an aging-related selective reduction in DAT gene expression in dopaminerigc neurons. Future studies are necessary to determine the significance of this result.

\section{The Subfamily of Glycine and Proline Transporters}

Several recent reports describe the cloning and functional expression of CDNAs that code for glycine transporters (47-49, 79). Two distinct glycine transporters, GLYT-1 and GLYT-2, that are identical except for the first 15 amino acid residues, appear to arise from a common gene by tissue-specific alternative splicing or alternative promotor usage (49). Northern blot analysis using oligonucleotide probes specific for each transporter demonstrated the differential expression of GLYT-1 and GLYT-2 in brain and peripheral tissues (49). Expression of GLYT-1 is widely distributed in brain but not in peripheral tissues. In contrast, GLYT-2 is expressed in brain and a number of peripheral tissues including spleen, lung, liver, stomach, uterus, and pancreas and in the RBL cell line. Using subtype-specific in situ hybridization probes, Borowsky and colleagues (49) observed nonoverlapping patterns of expression of GLYT-1 and GLYT-2 in rat brain. Expression of GLYT1 was found almost exclusively over white matter fiber tracts (implying expression in oligodendrocytes), whereas GLYT-2 exhibited a heterogeneous expression pattern within gray matter, paralleling the distribution of mRNA for the $\alpha-1$ and $\beta$-glycine receptor subunits. Thus, there appears to be a considerably wider distribution of glycine transporter mRNAs in brain than glycinergic neurons (reviewed in ref. 49).

Our understanding of the neural function(s) of glycine continues to evolve. In addition to its role as an inhibitory neurotransmitter in the spinal cord, brain stem, and retina, and its numerous metabolic functions, recent evidence suggests that glycine may also function as a neurotransmitter/modulator in more rostral brain regions (49). For example, glycine modulates excitatory transmission through a coagonist action at $\mathrm{N}$-methyl-Daspartate receptors (50). Future studies of the physiological role(s) of the individual GLYT subtypes may advance our understanding of the neural roles of glycine. These studies will also aid in the development of novel pharmacological agents with anticonvulsant, sedative, antiepileptic, and antispastic effects. The unexpected expression of the glycine transporter subtype 
GLYT-2 in mast cells and macrophages raises the possibility that glycine may play an important physiological role in these cells (49).

L-Proline has been postulated to be a neurotransmitter or neuromodulator in the mammalian CNS (reviewed in ref. 51). Like the well characterized amino acid neurotransmitters, including the excitatory amino acids, Lglutamate and L-aspartate, and the inhibitory amino acids GABA and glycine, rat brain synaptosomes and brain slices exhibit high affinity, $\mathrm{Na}^{+}$-dependent L-proline uptake (52). Furthermore, exogenously-loaded radiolabeled L-proline is released from brain slices in a $\mathrm{Ca}^{2+}$-dependent manner after $\mathrm{K}^{+}$-induced depolarization (53). Studies to define the role of L-proline in discrete pathways in the mammalian CNS have not been possible due to the inability to block its biosynthesis or high affinity transport in nervous tissue. Our recent cloning of a high affinity $\mathrm{Na}^{+}$-dependent $L$-proline transporter (rPROT) expressed in subpopulations of putative glutamatergic neurons of rat brain provides compelling support for a specific role for L-proline in excitatory transmission (51). The kinetic and pharmacological properties of rPROT clearly distinguish it from the low affinity widely expressed iminoglycine transporter (Table 1).

Glutamatergic synapses have been implicated in diverse physiological processes including the acquisition of associative learning, synapse formation, and neuroendocrine regulation. Abnormalities of glutamatergic neurotransmission have been implicated in the pathophysiology of excitotoxic neurological disorders. Thus an understanding of the physiological role(s) of the L-proline transporter, and its substrate, in specific glutamatergic neurons could provide novel insights into synaptic regulatory mechanisms involved in synaptic plasticity and excitotoxic neurological disorders. As a prelude to studies of expression of the L-proline transporter in human neurological disease, we have recently isolated CDNAs for the human homolog of this transporter from a human hippocampal CDNA library. These cDNA's exhibit approximately $92 \%$ nucleotide identity, and greater than $98 \%$ amino acid identity with rPROT

Table 1. Pharmacological Differences Between Rat Brain L-Proline Transporter and Brush Border Imino Transporter

\begin{tabular}{lcc}
\hline \multirow{2}{*}{ Compound } & \multicolumn{2}{c}{$\begin{array}{c}\text { Apparent inhibition constant } \\
(\mu \mathrm{M})^{*}\end{array}$} \\
\cline { 2 - 3 } & Rat brain $^{\circ}$ & Brush border $^{c}$ \\
\hline L-Proline & 9.7 & 300 \\
L-Pipecolate & 14 & 200 \\
Sarcosine (N-methylglycine) & 30 & 8700 \\
3,4-Dehydroproline & 31 & 2200 \\
L-Proline methyl ester & $>100$ & 400 \\
4-OH-L-Proline & $>100$ & 500 \\
\hline
\end{tabular}

- Inhibition constant values reflect mean estimates of triplicate determinations of uptake inhibition curves, adjusting for substrate concentration as described by Cheng and Prusoff (70).

${ }^{b} \mathrm{~T}_{7}$, vaccinia virus transient expression in HeLa cells (54).

' Rabbit jejunal brush border membrane vesicles (71).
(54). We have recently determined that expression of the human high affinity L-proline transporter (hPROT) is brain specific (54). Northern blot analysis revealed a prominent approximately 4-kb transcript in human brain, while no specific hybridizing species were detected in human liver, kidney, lung, placenta, skeletal muscle, heart, or pancreas. This apparent brain-specific expression of hPROT is particularly interesting, because other members of this family of neurotransmiter transporters do not show this degree of brain specificity. These results further underscore the potential relevance of the high affinity L-proline transporter in neural function. Studies of the expression and/or regulation of hPROT may shed light on the pathophysiology of type II hyperprolinemia, a genetic disorder characterized by elevated cerebrospinal fluid concentrations of L-proline and a high incidence of childhood seizures (55).

\section{Orphan Clones}

Two distinct CDNAs have been described that code for predicted proteins that exhibit amino acid sequence similarity to the family of $\mathrm{Na}^{+}$- (and $\mathrm{Cl}^{-}$)-dependent plasma membrane transporters but that exhibit distinct membrane topographies $(56,57)$. In addition to the large glycosylated extracellular loop separating transmembrane 3 and 4 , which these transporters share with the $\mathrm{Na}^{+}-$(and $\mathrm{Cl}^{-}$)-dependent transporters, they also contain a large presumably extracellular glycosylated loop separating transmembrane domains 7 and 8 (Fig. 1). This feature also makes these putative transporters approximately 730 amino acids long, about $10 \%$ longer than the other members of the $\mathrm{Na}^{+}$(and $\mathrm{Cl}^{-}$)dependent transporter family. Sequence comparisons show that these orphan clones exhibit approximately $65 \%$ amino acid sequence identity to each other, but only approximately $35 \%$ amino acid sequence identity with the other members of the $\mathrm{Na}^{+}-\left(\right.$and $\mathrm{Cl}^{-}$)-dependent transporter family $(56,57)$.

Functional expression studies of the orphan clones have failed to identify their substrates. However, it is unclear whether the putative orphan transporters are being improperly expressed or whether the appropriate substrates have not yet been identified. The development of epitope-tagged orphan constructs and/or the development of specific antibodies directed against the predicted orphan proteins should aid in interpretation of future expression studies. The apparent brain-specific expression of the cognate mRNAs for the orphan cDNAs $(56,57)$ indicate that these putative transporters may be performing interesting roles in nervous tissue.

\section{$\mathrm{Na}^{+}$- (and $\mathrm{K}^{+}$)-Dependent Glutamate Transporters}

The active transport of glutamate into neurons and glial cells plays a key role in regulating synaptic and extracellular concentrations of this excitatory neurotransmitter. Recently, three distinct cDNA clones encoding structurally related (49-56\% sequence identity) high affinity L-glutamate transporters have been described. 
Storck et al. (58) were the first to describe the cloning of a high affinity glutamate/aspartate transporter, which they called GLAST-1. During the purification of an enzyme involved in cerebroside biosynthesis they copurified a hydrophobic protein of 66 kilodaltons. Using synthetic oligonucleotide probes based upon partial amino acid sequences of the purified protein, they isolated CDNA clones from a rat brain cDNA library. Nucleotide sequence analysis revealed significant sequence similarity to prokaryotic proton-dependent glutamate/aspartate carriers (59). Functional expression of the cDNA in Xenopus oocytes induced high affinity L-glutamate transport. Subsequently two additional glutamate transporter CDNAs were described. Pines et al. (60) used antibodies directed against a glutamate transporter purified from rat brain to isolate a CDNA which they called GLT-1. GLT-1 immunoreactivity had previously been localized to astrocytic processes by immunocytochemistry (61). Expression of GLT-1 CDNA in HeLa cells induced high affinity glutamate uptake that was $\mathrm{Na}^{+}\left(\right.$and $\left.\mathrm{K}^{+}\right)$dependent but $\mathrm{Cl}^{-}$independent. Kanai and Hediger (62) isolated a glutamate transporter, which they called EAAC-1, from a rabbit intestine CDNA library by expression cloning with Xenopus oocytes.

Figure 1 depicts a tentative structural model of the glutamate transporters. Because of the subjective nature of hydropathy analysis, ambiguities exist in the assignment of the number of proposed transmembrane domains of the three cloned glutamate transporters ( 58 , $60,62)$. Six transmembrane-spanning domains can be identified in the amino-terminal approximately 340 amino acids of the glutamate transporters, while from two to six transmembrane-spanning domains can be predicted in the carboxy-terminal amino acids $(-340$ to carboxy-terminus). Arriza et al. (63) have previously noted that many of the hydrophobic regions predicted by sequence hydropathy analysis within the glutamate transporter family are more consistent with $\beta$-sheets than $\alpha$-helices. Future studies are necessary to experimentally establish the membrane topography of the glutamate transporters.

Two of the glutamate transporter subtypes, GLAST1 and GLT-1, are expressed predominantly in brain. GLT-1. is widely expressed throughout the CNS in astrocytes (60), while GLAST-1 is expressed at high levels in Bergmann glial cells in the cerebellum (58). In contrast, EAAC-1 is expressed in brain as well as a number of peripheral tissues including liver, kidney, and intestine (62). Surprisingly, within the brain, EAAC-1 is expressed in neurons, with prominent expression in glutamatergic hippocampal pyramidal and granule neurons (60). Future studies of the cellular and subcellular localization of the cloned glutamate transporter subtypes are necessary to provide insights into potential roles that individual subtypes might play in the regulation of excitatory amino acid neurotransmission. In particular, it will be important to establish which glutamate transporter subtype(s) is (are) expressed in glutamatergic nerve terminals.

An $\mathrm{Na}^{+}$-independent, $\mathrm{Cl}^{-}$-dependent L-glutamate transport activity has been described in rat brain synaptosomes (64) and glioma cell lines (65). Based upon differences in ionic requirements, compared to the cloned glutamate transporters, it is likely that this glutamate transporter belongs to a different family of membrane transport proteins. Because abnormalities in glutamate transport have been implicated in excitotoxic neurological disorders, it will be important to determine the extent of the molecular diversity of the family of glutamate transporters and the physiological roles of the individual subtypes.

Previously, a human-expressed sequence tag was described that exhibited distant homology to an Escherichia coli glutamate/aspartate carrier (66). To explore the diversity within the family of glutamate transporters, we performed a PCR on human hippocampal poly $(A)^{+}$ RNA using primers corresponding to this sequence. We identified a novel PCR product that was used to isolate a full-length cDNA clone, which we called SATT, from human hippocampus that exhibited significant homology with mammalian glutamate transporters (67). Surprisingly, despite the sequence relationship with glutamate transporters, expression of this CDNA clone in HeLa cells did not induce glutamate transport but did induce $\mathrm{Na}^{+}$-dependent neutral amino acid uptake with several properties similar to the $\mathrm{Na}^{+}$-dependent neutral amino acid transport activity designated system ASC (reviewed in ref. 67).

A comparison of the amino acid sequence of SATT with those of the three cloned mammalian glutamate transporters may offer insights into the structure/function properties of these structurally related but functionally distinct transporters (Fig. 4). Because SATT does not transport glutamate, those amino acid motifs that it shares with the glutamate transporters, such as the $A A(I, V, L) F I A Q$ motif that is conserved throughout the evolutionary diversity of glutamate transporters from prokaryotes to mammals, may be involved in common functions that these transporters share, such as the formation and maintenance of a functional transporter. These shared motifs are unlikely to be involved in substrate recognition. In contrast, those amino acid residues that are conserved in the glutamate transporters, but absent in SATT, could play a role in substrate recognition and/or unique regulatory properties of the glutamate transporters. In this respect, a number of polar and charged residues are conserved between the mammalian glutamate transporters but are substituted by amino acids that are either neutral or have an opposite charge in SATT (Fig. 4). For example, a conserved arginine residue in the glutamate transporters $\left(R_{477}\right.$ of GLT-1 in Fig. 4) is replaced by threonine in the neutral amino acid carrier SATT. Future site-directed mutagenesis studies will examine the possibility that these residues play a role in glutamate recognition and/ or translocation. Previously it has been demonstrated that system ASC can transport anionic amino acids at $\mathrm{pH}$ less than $6(68)$. Future studies will characterize the $\mathrm{pH}$ dependence of the transport properties of SATT to investigate the functional basis for the structural and 


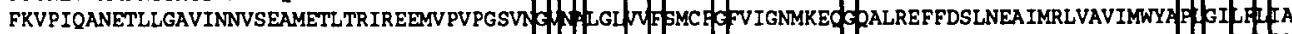

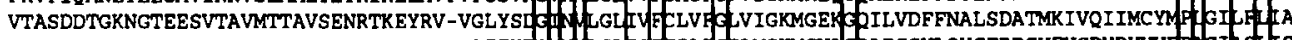

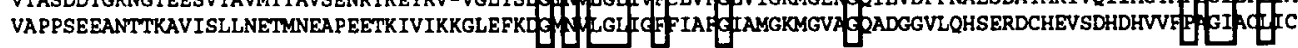

Fig. 4. Alignment of the predicted amino acid sequences of the $\mathrm{Na}^{+}$- (and $\mathrm{K}^{+}$)-dependent, $\mathrm{Cl}^{-}$-independent plasma membrane transporter gene family. SATT is the human $\mathrm{Na}^{+}$-dependent neutral amino acid transporter, which exhibits significant amino acid sequence similarity with mammalian plasma membrane glutamate transporters (67). GLAST1 is the rat brain glutamate transporter described by Storck et al. (58). EAAC1 is glutamate transporter isolated by Kanai and Hediger (62) from a rabbit small intestine cDNA library. GLT1 is the rat brain glutamate transporter isolated by Pines et al. (60). Boxed residues represent those amino acids conserved across all four transporters.

sequence similarity between SATT and the mammalian $\mathrm{Na}^{+} /$glutamate cotransporters. The extent of the molecular diversity of the glutamate transporter family and perhaps of a larger $\mathrm{Na}^{+}$- (and $\mathrm{K}^{+}$)-dependent, $\mathrm{Cl}^{-}$-independent transporter gene family remains to be established.

\section{Proton-Dependent Synaptic Vesicle Transporters}

Synaptic vesicle neurotransmitter transporters represent a third family of membrane transporters crucial for chemical transmission at synapses (see ref. 69 for review). These carriers transport neurotransmitters from the cytoplasm into the synaptic vesicle lumen using the energy of a proton gradient; uptake of the neurotransmitter into the vesicle is coupled to the extrusion of a proton into the cytoplasm. At least four distinct transport activities have been identified using purified synaptic vesicles: one for dopamine, serotonin, and norepinephrine, a second for the excitatory amino acid glutamate, a third for acetylcholine, and a fourth for GABA and glycine (69). Recently, significant progress has been made in the molecular characterization of synaptic vesicle neurotransmitter transporters. Two distinct monoamine vesicle transporters have been cloned: a chromaffin granule amine transporter and a highly related transporter expressed in catecholaminergic neurons of the brain stem and midbrain $(72,73)$. Hydropathy analysis of the predicted proteins predicts the presence of 12 transmembrane-spanning domains and a lumenal loop separating transmembrane domains 1 and 2. Recently, the unc-17 gene from Caenorhabditis elegans has been cloned (74). Unc-17 mutations in C. elegans produce deficits in neuromuscular function (reviewed in ref. 74). On the basis of sequence similarity to the mammalian vesicular monoamine transporters and of localization to synaptic vesicles of cholinergic neurons in C. elegans, the unc-17 gene product appears to encode a vesicular transporter for acetylcholine (74). Based upon the amino acid sequence homology between the mammalian vesicular monoamine transporters and the putative $C$. elegans synaptic vesicle acetycholine transporter, the rapid identification of additional synaptic vesicle neurotransmitter transporters can be expected.

\section{FUTURE DIRECTIONS}

The molecular identification of neurotransmitter transporters should permit a refined analysis of the physio- 
logical role of neurotransmitter uptake in synaptic transmission at chemical synapses. Historically, the role that neurotransmitter transporters play in the regulation of synaptic transmission has been inferred from the dramatic behavioral effects of drugs that block biogenic amine transport. However, the precise influence of neurotransmitter transport on synaptic efficacy is not known and the role of uptake regulation in the modulation of synaptic strength remains largely speculative. Does neurotransmitter reuptake differentially modulate synaptic responses at metabotropic vs. ionotropic receptors? The development of a cell culture system where presynaptic reuptake currents and related postsynaptic responses can be studied after $\mathrm{Ca}^{2+}$-evoked transmitter release indicates that answers should be forthcoming (80). The dramatic progress made in the molecular cloning and characterization of neurotransmitter transporters will provide molecular, genetic, and immunological tools with which to explore the synaptic role of neurotransmitter transport. Specific transporters can now be expressed in isolation and their pharmacological and biophysical (75-77) properties studied. Furthermore, it should now be possible to elucidate binding sites for psychoactive drugs through chimeric transporter and site-directed mutagenesis studies. Specific antibodies can be prepared against particular transporter proteins for immunocytochemical localization of the cellular and subcellular distribution of transporters. These antibodies should permit studies of the mechanisms that neurons use to target transporter proteins to synapses. An examination of the physiological basis for multiple transporter subtypes for a given neurotransmitter may reveal new levels of synaptic heterogeneity. Moreover, the existence of neurotransmitter transporter subtypes should aid in the development of new therapeutic agents with reduced side effect profiles for treating neuropsychiatric disorders. The availability of transporter cDNAs and genes (78) will also permit an examination of the potential role of transporter defects in neuropsychiatric disorders. Finally, as the molecular diversity of neurotransmitter transporters continues to be explored, the discovery of the substrates for novel transporters may identify new molecules with synaptic regulatory roles. The ability to breed transgenic animals in which specific transporter genes have been overexpressed or inactivated by homologous recombination, may prove particularly powerful in defining the role of novel transporters in the nervous system. 1993.

Received September 8, 1993. Accepted September 10,

Address requests for reprints to: Dr. Robert T. Fremeau, Jr., Box 3813, 436 Nanaline H. Duke Building, Duke University Medical Center, Durham, North Carolina 27710.

\section{REFERENCES}

1. Amara SG, Kuhar MJ 1993 Neurotransmitter transporters: recent progress. Annu Rev Neurosci 16:73-93
2. Axelrod J 1971 Noradrenaline: fate and control of its biosynthesis. Science 173:598-606

3. Snyder SH 1991 Vehicles of inactivation. Nature 354:187

4. Kanner BI, Schuldiner S 1987 Mechanism of transport and storage of neurotransmitters. CRC Crit Rev Biochem 22:1-38

5. Kuhar MJ, Murrin LC 1978 Sodium-dependent, high affinity choline uptake. J Neurochem 30:15-21

6. Snyder SH 1970 Putative neurotransmitters in the brain: selective neuronal uptake, subcellular localization, and interactions with centrally acting drugs. Biol Psychiatry 2:367-389

7. Nicholls D, Attwell D 1990 The release and uptake of excitatory amino acids. TIPS 11:462-468

8. Krogsgaard-Larsen Falch E, Larsson OM, Schousboe A 1987 GABA uptake inhibitors: relevance to antiepileptic drug research. Epilepsy Res 1:77-93

9. Snyder SH, D'Amato RJ 1986 MPTP: a neurotoxin relevant to the pathophysiology of Parkinson's disease. Neurology 36:250-258

10. Guastella J, Nelson N, Nelson H, Czyzyk L, Keynan S, Miedel MC, Davidson N, Lester HA, Kanner BI 1990 Cloning and expression of a rat brain GABA transporter. Science 249:1303-1306

11. Pacholczyk T, Blakely RD, Amara SG 1991 Expression cloning of a cocaine- and antidepressant-sensitive human noradrenaline transporter. Nature 350:350-354

12. Henderson PJ 1990 The homologous glucose transport proteins of prokaryotes and eukaryotes. Res Microbiol 141:316-328

13. Hediger MA, Coady MJ, Ikeda TS, Wright EM 1987 Expression cloning and cDNA sequencing of the $\mathrm{Na}^{+} /$ glucose cotransporter. Nature 330:379-381

14. Yazyu $H$, Shiota-Niiya $S$, Shimamoto $T$, Kanazawa $H$, Futai M, Tsuchiya T 1984 Nucleotide sequence of the $m e / B$ gene and characteristics of deduced amino acid sequence of the melibiose carrier in Escherichia coli. J Biol Chem 259:4320-4326

15. Endicott JA, Ling V 1989 The biochemistry of P-glycoprotein-mediated multidrug resistance. Annu Rev Biochem 58:137-171

16. Riordan JR, Rommens JM, Kerem B-S, Alon N, Rozmahel R, Grzelczak Z, Zielinski J, Lok S, Plavsic N, Chou J-L, Drumm ML, lannuzzi MC, Collins FS, Tsui L-C 1989 Identification of the cystic fibrosis gene: cloning and characterization of complementary DNA. Science 245:10661065

17. Clark JA, Deutch AY, Gallipoli PZ, Amara SG 1992 Functional expression and CNS distribution of a $\beta$-alaninesensitive neuronal GABA transporter. Neuron 9:337-348

18. Borden LA, Smith KE, Hartig PR, Branchek TA, Weinshank RL 1992 Molecular heterogeneity of the $\gamma$-aminobutyric acid (GABA) transport system: Cloning of two novel high affinity GABA transporters from rat brain. $J$ Biol Chem 267:21098-21104

19. Liu Q-R, Lopez-Corcuera B, Mandiyan S, Nelson H, Nelson N 1993 Molecular characterization of four pharmacologically distinct $\gamma$-aminobutyric acid transporters in mouse brain. J Biol Chem 268:2106-2112

20. Pantanowitz S, Bendahan A, Kanner BI 1993 Only one of the charged amino acids located in the transmembrane $\alpha$-helices of the $\gamma$-aminobutyric acid transporter (subtype A) is essential for activity. J Biol Chem 268:3111-3225

21. Yamauchi A, Uchida S, Kwon HM, Preston AS, Robey RB, Garcia-Perez A, Burg MB, Handler JS 1992 Cloning of a $\mathrm{Na}^{+}$- and $\mathrm{Cl}^{-}$-dependent betaine transporter that is regulated by hypertonicity. J Biol Chem 267:649-652

22. Uchida S, M, Kwon HM, Yamauchi A, Preston AS, Maruma F, Handler JS 1992 Molecular cloning of the $c$ DNA for an MDCK cell $\mathrm{Na}^{+}$- and $\mathrm{Cl}^{-}$-dependent taurine transporter that is regulated by hypertonicity. Proc Natl Acad Sci USA 89:8230-8234

23. Smith KE, Borden LA, Wang CH, Hartig PR, Branchek TA, Weinshank RL 1992 Cloning and expression of a high 
affinity taurine transporter from rat brain. Mol Pharmacol 42:563-569

24. Liu QR, Lopez-Corcuera B, Nelson H, Mandiyan S, Nelson $N 1992$ Cloning and expression of a cDNA encoding the transporter of taurine and $\beta$-alanine in mouse brain. Proc Natt Acad Sci USA 89:12145-12149

25. Jhiang SM, Fithian L, Smanik P, McGill J, Tong Q, Mazzaferri EL 1993 Cloning of the human taurine transporter and characterization of taurine uptake in thyroid cells. FEBS Lett 318:139-144

26. Sturman JA 1990 Taurine deficiency. Prog Clin Blol Res 351:385-395

27. Guimbal C, Kiliman MW $1993 \mathrm{~A} \mathrm{Na}^{+}$-dependent creatine transporter in rabbit brain, muscle, heart, and kidney. $J$ Biol Chem 268:8418-8421

28. Bradley CC, Moore KR. Fremeau Jr RT, Ramamoorthy S, Ganapathy V, Han H, Yang-Feng T, Blakely RD 1993 Molecular cloning, expression, and chromosomal localization of a rat brain creatine transporter. Soc Neurosci Abstr $19: 220$

29. Shimada S, Kitayama S, Lin C-L, Patel A, Nanthakumar E, Gregor P, Kuhar M, Uhl G 1991 Cloning and expression of a cocaine-sensitive dopamine transporter complementary DNA. Science 254:576-578

30. Kilty JE, Lorang D, Amara SG 1991 Cloning and expression of a cocaine-sensitive rat dopamine transporter. Science 254:578-579

31. Usdin TB, Mezey E, Chen C, Brownstein MJ, Hoffman BJ 1991 Cloning of the cocaine-sensitive bovine dopamine transporter. Proc Natl Acad Sci USA 88:11168-11171

32. Giros B, El Mestikawy S, Godinot N, Zheng K, Han H, Yang-Feng T, Caron MG 1992 Cloning, pharmacological characterization, and chromosome assignment of the human dopamine transporter. Mol Pharmacol 42:383-390

33. Vandenbergh DJ, Persico AM, Uhl GR 1992 A human dopamine transporter cDNA predicts reduced glycosylation, displays a novel repetitive element and provides racially-dimorphic Taq1 RFLPs. Mol Brain Res 15:161166

34. Blakely RD, Berson HE, Fremeau Jr RT, Caron MG, Peek MM, Prince HK, Bradley CC 1991 Cloning and expression of a functional serotonin transporter from rat brain. Nature 354:66-70

35. Hoffman BJ, Mezey E, Brownstein MJ 1991 Cloning of a serotonin transporter affected by antidepressants. Science 254:579-580

36. Ramamoorthy S, Bauman AL, Moore KR, Han H, YangFeng T, Chang AS, Ganapathy V, Blakely RD 1993 Antidepressant- and cocaine-sensitive human serotonin transporter: molecular cloning, expression, and chromosomal localization. Proc Natl Acad Sci USA 90:2542-2546

37. Hinson JP, Vinson GP, Pudney J, Whitehouse BJ 1989 Adrenal mast cells modulate vascular and secretory responses in the intact adrenal gland of the rat. Endocrinology 121:253-260

38. Holzwarth MA, Brownfield MS 1985 Serotonin coexists with epinephrine in rat adrenal medullary cells. Neuroendocrinology 41:230-236

39. Cerruti C, Walther DM, Kuhar MJ, Uhl GR 1993 Dopamine transporter $m$ RNA expression is intense in rat midbrain neurons and modest outside midbrain. Mol Brain Res 18:181-186

40. Myers CL, Lazo JS, Pitt BR 1989 Translocation of protein kinase $C$ is associated with inhibition of 5-HT uptake by cultured endothelial cells. Am J Physiol 257:L253-L258

41. Cool DR, Leibach FH, Bhalla VK. Mahesh VB, Ganapathy $\checkmark 1991$ Expression and cyclic-AMP-dependent regulation of a high affinity serotonin transporter in the human placental choriocarcinoma cell line (JAR). J Biol Chem 266:15750-15757

42. Javitch JA, D'Amato RJ, Strittmatter SM, Snyder S 1985 Parkinsonism-inducing neurotoxin, $N$-methyl-4-phenyl1,2,3,6-tetrahydropyridine: uptake of the metabolite $\mathrm{N}$ - methyl-4-phenylpyridine by dopamine neurons explains selective toxicity. Proc Natl Acad Sci USA 82:2173-2177

43. Amara SG, Arriza JL 1993 Neurotransmitter transporters: three distinct gene families. Curr Opin Neurobiol 3:337344

44. Turk E, Zabel B, Mundlos S, Dyer J, Wright EM 1991 Glucose/galactose malabsorption caused by a defect in the $\mathrm{Na}^{+}$/glucose cotransporter. Nature 350:354-356

45. Kitayama S, Shimada S, Xu H, Markham L, Donovan DM, Uhl GR 1992 Dopamine transporter site-directed mutations differentially alter substrate transport and cocaine binding. Proc Natl Acad Sci USA 89:7772-7785

46. Bannon MJ, Poosch MS, Xia Y, Goebel DJ, Cassin B, Kapatos G 1992 Dopamine transporter mRNA content in human substantia nigra decreases precipitously with age. Proc Natl Acad Sci USA 89:7095-7099

47. Smith KE, Borden LA, Hartig PR, Branchek T, Weinshank RL 1992 Cloning and expression of a glycine transporter reveal colocalization with NMDA receptors. Neuron 8:927-935

48. Guastella J, Brecha N, Weigmann C, Lester H, Davidson $N 1992$ Cloning, expression, and localization of a rat brain high-affinity glycine transporter. Proc Natl Acad Sci USA 89:7189-7193

49. Borowsky B, Mezey E, Hoffman BJ 1993 Two glycine transporter variants with distinct localization in the CNS and peripheral tissues are encoded by a common gene. Neuron 10:851-863

50. Johnson JW, Ascher P 1987 Glycine potentiates the NMDA response in cultured mouse brain neurons. Nature 325:529-531

51. Fremeau Jr RT, Caron MG, Blakely RD 1992 Molecular cloning and expression of a high affinity L-proline transporter expressed in putative glutamatergic pathways of rat brain. Neuron 8:915-926

52. Bennett Jr JP, Logan WJ, Snyder SH 1972 Amino acid neurotransmitter candidates: sodium-dependent high-affinity uptake by unique synaptosomal fractions. Science 178:997-999

53. Bennett JP, Mulder AH, Snyder SH 1974 Neurochemical correlates of synaptically active amino acids. Life Sci 15:1045-1056

54. Fremeau Jr RT, Shafqat S, Yang-Feng T 1993 Molecular cloning and chromosomal localization of a human brainspecific L-proline transporter. Soc Neurosci Abstr 19:220

55. Phang JM, Scriver CR 1989 Disorders of proline and hydroxyproline metabolism. In: Scriver CR, Beaudet AL, Shy WS, Valle D (eds) The Metabolic Basis of Inherited Disease, ed 6. McGraw-Hill, New York, pp 577-597

56. Uhl GR, Kitayama S, Gregor P, Nanthakumar E, Persico A, Shimada S 1992 Neurotransmitter transporter family cDNAs in a rat brain midbrain library: "orphan transporters" suggest sizable structural variations. Mol Brain Res 16:353-359

57. Liu Q-R, Mandiyan S, Lopez-Corcuera B, Neslon $\mathrm{H}$, Nelson N 1993 A rat brain cDNA encoding a neurotransmitter transporter with an unusual structure. FEBS Lett 315:114-118

58. Storck T, Schulte S, Hoffman K, Stoffel WH 1992 Structure, expression, and functional analysis of a $\mathrm{Na}^{+}$-dependent glutamate/aspartate transporter from rat brain. Proc Natl Acad Sci USA 89:10855-10859

59. Tolner B, Poolman B, Wallace B, Konings WN 1992 Revised nucleotide sequence of the glt $P$ gene, which encodes the proton-glutamate-aspartate transport protein of Escheichia coli K-12. J Bacteriol 174:2391-2393

60. Pines G, Danbolt NC, Bjoras M, Zhang Y, Bendahan A, Eide L Koepsell H, Storm-Mathisen J, Seeberg E, Kanner BI 1992 Cloning and expression of a rat brain L-glutamate transporter. Nature 360:464-467

61. Danbolt NC, Storm-Mathisen J, Kanner BI $1992 \mathrm{~A}\left[\mathrm{Na}^{+}+\right.$ $\left.\mathrm{K}^{+}\right]$-coupled $\mathrm{L}$-glutamate transporter purified from rat brain is located in glial cell processes. Neuroscience 51:295310 
62. Kanai $Y$, Hediger M 1992 Primary structure and functional characterization of a high affinity glutamate transporter. Nature 360:467-471

63. Arriza JL, Kavanaugh MP, Fairman W, Wu Y-N, Murdoch GH, North RA, Amara SG 1993 Cloning and expression of a human neutral amino acid transporter with structural similarity to the glutamate transporter gene family. J Biol Chem 268:15329-15332

64. Zaczek R, Balm M, Arlis S, Drucker H, Coyle JT 1987 Quisqualate-sensitive, chloride-dependent transport of glutamate into rat brain synaptosomes. J Neurosci Res 18:425-431

65. Waniewski RA, Martin DL 1984 Characterization of Lglutamic acid transport by glioma cells in culture, evidence for sodium-independent, chloride-dependent high affinity influx. J Neurosci 4:2237-2246

66. Adams MD, Dubnick M, Kerlavage AR, Moreno R, Kelley JM, Utterback TR, Nagle JW, Fields C, Venter JC 1992 Sequence identification of 2,375 human brain genes. Nature 355:632-634

67. Shafqat S, Tamarappoo BK, Kilberg MS, Puranam RS, MCNamara JO, Guadano-Ferraz A, Fremeau Jr RT 1993 Cloning and expression of a novel $\mathrm{Na}^{+}$-dependent neutral amino acid transporter structurally related to mammalian $\mathrm{Na}^{+} /$glutamate cotransporters. J Biol Chem 268:1535115355

68. Vadgamma JV, Christensen HN 1984 Wide distribution of pH-dependent service of transport system ASC for both anionic and zwitterionic amino acids. J Biol Chem 259:3648-3652

69. Edwards RH 1992 The transport of neurotransmitters into synaptic vesicles. Curr Opin Neurobiol 2:586-594

70. Cheng Y-C, Prusoff WH 1973 Relationship between the inhibition constant $(\mathrm{Ki})$ and the concentration of inhibitor which causes 50 percent inhibition $\left(I_{50}\right)$ of an enzymatic reaction. Biochem Pharmacol 22:3099-3108

71. Stevens BR, Kaunitz JD, Wright EM 1984 Intestinal trans- port of amino acids and sugars: advances using membrane vesicles. Annu Rev Physiol 46:417-433

72. Liu Y, Peter D, Roghani A, Schuldiner S, Prive GG, Eisenberg D, Brecha N, Edwards RH 1992 A cDNA that suppresses MPP+ toxicity encodes a vesicular amine transporter. Cell 70:539-551

73. Erickson JD, Eiden LE, Hoffman BJ 1992 Expression cloning of a reserpine-sensitive vesicular monoamine transporter. Proc Natl Acad Sci USA 89:10993-10997

74. Alfonso A, Grundahl K, Duerr JS, Han H-P, Rand JB 1993 The Caenorhabditis elegans unc-17 gene: a putative vesicular acetylcholine transporter. Science 261:617-619

75. Kavanaugh MP, Arriza JL, North RA, Amara SG 1992 Electrogenic uptake of $\gamma$-aminobutyric acid by a cloned transporter expressed in Xenopus oocytes. J Biol Chem 267:22007-22009

76. Mager S, Naeve J, Quick M, Labarca C, Davidson N, Lester HA 1993 Steady states, charge movements and rates for a cloned GABA transporter expressed in Xenopus oocytes. Neuron 10:177-188

77. Klockner U, Storck T, Conradt M, Stoffel W 1993 Electrogenic L-glutamate uptake in Xenopus laevis oocytes expressing a cloned rat brain L-glutamate/L-aspartate transporter (GLA ST-1). J Biol Chem 268:14594-14596

78. Liu Q-R, Mandiyan S, Nelson H, Nelson N 1992 A family of genes encoding neurotransmitter transporters. J Biol Chem 89:6639-6643

79. Liu Q-R, Nelson H, Mandiyan S, Lopez-Corcuera B, Nelson $N 1992$ Cloning and expression of a glycine transporter from mouse brain. FEBS Lett 305:110-114

80. Bruns D, Engert F, Lux H-D 1993 A fast activating presynaptic reuptake current during serotonergic transmission in identified neurons of Hirudo. Neuron 10:559-572

81. Lopez-Corcuera B, Liu Q-R, Mandiyan S, Nelson H, Nelson N 1992 Expression of a mouse brain cDNA encoding novel $\gamma$-aminobutric acid transporter. J Biol Chem 267:17491-17493 